\title{
Effect of Acute Aerobic Exercise on Inhibition of Motor Response Based on Correlation Analysis
}

\author{
Junying Zhu', Xia Liu* \\ Department of Physical Education, Ocean Univercity of China, Qingdao 266100, Shandong, China \\ *Corresponding Author.
}

\begin{abstract}
:
In this paper, the effects of acute moderate intensity aerobic exercise on inhibition ability of college students were investigated from different aspects, such as behavioral performance, physiological response, and cognitive processing mode of brain. In this paper, college students were selected as the research object, and the content of brain-derived neurotrophic factor (BDNF) in blood was recorded and analyzed after moderate intensity aerobic exercise or reading intervention at the same time. In this paper, we completed the behavioral response and brain activity of the adapted go / NoGo task. After acute moderate intensity aerobic exercise, the correct rate of behavior inhibition of college students increased. The content of BDNF increased, and Nogo-N2 and NoGo-P3, which represent the inhibition ability, had obvious effect. Acute moderate intensity aerobic exercise can effectively promote the inhibition ability of college students, and its mechanism may lie in the rapid recognition of inhibition signals and the allocation of cognitive resources, as well as more peripheral BDNF circulation.
\end{abstract}

Keywords: Acute Aerobic Exercise, Inhibition Ability, Brain-derived Neurotrophic Factor (BDNF), EEG Components.

\section{INTRODUCTION}

With the deepening of scientific research, scholars have found that moderate intensity aerobic exercise can promote cognitive function. Inhibition control, as the core component of cognitive function, has been widely studied [1-2]. Through questionnaires, cognitive tasks and other ways, researchers have explored behavioral response or electrophysiological response respectively, and obtained positive results from different levels [3]. However, there is still a lack of research on comprehensive investigation of behavioral response, physiological response and brain response, so as to get support from different levels of behavior to physiology [4-6].

In this study, ordinary college students were selected as the research object. By using the MS-Go / Nogo task, the effects of moderate intensity aerobic exercise on inhibition control ability were compared and analyzed from the aspects of behavior, physiology and electrophysiology. The purpose of this study is to provide theoretical support and scientific methods for aerobic exercise to promote inhibition control ability. 


\section{METHOD}

\subsection{Research objects}

Seventeen college students, aged 19-25, were selected. Among them, there were 8 males and 9 females. The criteria for selecting the subjects were as follows: (1) those who showed normal physical activity ability in the ipar-q questionnaire; (2) the body mass index (BMI) was 18.5-23.5; (3) those with normal intelligence assessed by raven's simple reasoning intelligence scale; (4) those with normal naked or corrected visual acuity. See Table 1.

TABLE I. Basic information of subjects

\begin{tabular}{|c|c|c|c|c|c|}
\hline $\begin{array}{c}\text { NUMBER OF } \\
\text { PEOPLE (N) }\end{array}$ & $\begin{array}{c}\text { GENDER } \\
\text { (MALE / } \\
\text { FEMALE) }\end{array}$ & $\begin{array}{c}\text { AGE } \\
\text { (YEARS) }\end{array}$ & IPARQ(METS) & BMI & $\begin{array}{c}\text { INTELLIGENCE } \\
\text { SCORE }\end{array}$ \\
\hline 17 & $8 / 9$ & $20.65 \pm 1.25$ & $412.32 \pm 109.64$ & $22.53 \pm 2.06$ & $46.35 \pm 4.45$ \\
\hline
\end{tabular}

\subsection{Measuring method}

\section{1) Determination of BDNF content}

The serum BDNF protein level was detected by double antibody sandwich ba-elisa [7-9]. The kit was purchased from R \& D company of USA. Human BDNF monoclonal antibody was coated on the bottom of 96 microplate, then standard, serum sample, biotinylated anti human BDNF antibody, horseradish peroxidase labeled streptavidin were added in turn, and incubated at room temperature for 2 hours to form antigen antibody complex and avidin biotin peroxidase complex. Then wash the plate, add the substrate TMB and incubate at room temperature for $30 \mathrm{~min}$, and finally add the termination solution to make it yellow. The degree of yellow is directly proportional to the concentration of BDNF in serum. The 0d absorbance value was measured at $570 \mathrm{~nm}$ of the microplate reader, and the serum BDNF concentration was calculated by drawing the standard curve.

2) Cognitive behavioral measurement

The cognitive task was selected from Ms go / NoGo paradigm. The experimental stimuli included two letters: $\mathrm{X}, \mathrm{y}$, and the stimulus presented random order. The subjects were asked to judge whether the present stimulus was the same as the previous one, that is, when the letter presented was different from the previous one (such as XY...), it was the go test, and the subjects were asked to press the " 0 ' key to react; if the letter presented was the same as the previous one (such as XX...)) The subjects were asked not to press the key (see Figure 1). The ratio of go trial to Nogo trial was $4: 1$. There were four blocks in this experiment, each block included 200 trials, a total of 800 trials. The frequency of each trial was $1 \mathrm{~Hz}$, the longest time of letter stimulation was $400 \mathrm{~ms}$, and the trial was finished by pressing the key. There is a 5 -minute break between blocks [10].

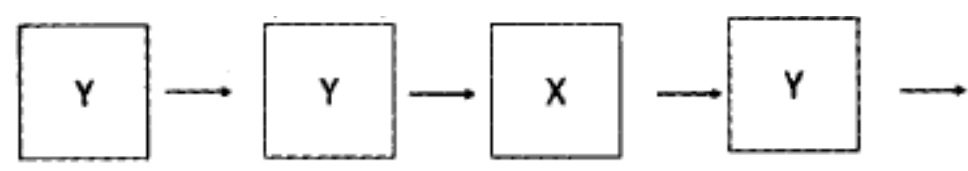

Fig 1: Experiment flow chart

\subsection{Research process}

In this experiment, 17 subjects were divided into two groups, and all subjects were required to complete the basic situation test, exercise intervention and reading intervention three times. The order of exercise intervention and reading intervention in the two groups was balanced between the subjects. The first test was 
the basic situation test. After arriving at the laboratory, the height and weight of the subjects were measured, and then ipar-q questionnaire and simple Raven reasoning intelligence scale were completed. After the screening of the above indicators, the selected subjects were tested twice after the grouping. In the third test 10 days later, the treatment schemes of the two groups were exchanged. This experiment is designed to eliminate the learning effect and order effect of the test. At the same time, the place and time of the two tests are consistent. See Table 2.

In the exercise intervention of the participants, the heart rate interval value of each participant in moderate intensity exercise was converted by the heart rate of 3 minutes resting state in the American Sports Medical Association (ACSM), so as to regulate and monitor exercise load in exercise. Combined with the relevant research of college students in China, the middle intensity exercise load of college students is set as the maximum heart rate of $60 \%-69 \%$ of the individual, in which the maximum heart rate is obtained by the formula HR $\max =220$ age.

\section{TABLE II. Inter subject balance in intra group crossover design}

\begin{tabular}{|c|c|c|c|}
\hline GROUP & $\begin{array}{c}\text { FOR THE FIRST } \\
\text { TIME }\end{array}$ & THE SECOND TIME & THIRD TIME \\
\hline GROUP A & $\begin{array}{c}\text { BASIC } \\
\text { INFORMATION }\end{array}$ & $\begin{array}{c}\text { EXERCISE } \\
\text { INTERVENTION }\end{array}$ & $\begin{array}{c}\text { READING } \\
\text { INTERVENTION }\end{array}$ \\
\hline (8 PERSONS) & TEST & (SPORT A) & (READING A) \\
\hline GROUP B & $\begin{array}{c}\text { BASIC } \\
\text { INFORMATION }\end{array}$ & $\begin{array}{c}\text { READING } \\
\text { INTERVENTION }\end{array}$ & $\begin{array}{c}\text { EXERCISE } \\
\text { INTERVENTION }\end{array}$ \\
\hline (9 PERSONS) & TEST & (READING B) & (SPORT B) \\
\hline
\end{tabular}

In the formal experimental stage, the subjects in the exercise intervention group completed all the EEG preparations, and then all the subjects were familiar with the cognitive tasks through the guidance language and practice. The exercise intervention group performed 30 minutes of moderate intensity aerobic exercise on the power bicycle (Monark 834, Sweden). The exercise intensity was monitored by heart rate meter (rs800cxsd, Finland) according to the heart rate interval value obtained in the basic situation test, and the reader's digest was read in the reading intervention group at the same time. At the end of exercise / reading, the cognitive task was completed on the de1119 inch display after the EEG instrument was connected. The task time is about 20 minutes. After the behavior test, blood was collected from elbow vein and frozen at $20{ }^{\circ} \mathrm{C}$ for biochemical analysis.

\subsection{Statistical analysis}

SPSS 17.0 was used for statistical analysis. First, the paired t-test was used to verify that the sequence effect of the crossover design within the group was negative, and then the data were further analyzed. Then, the intervention factors (exercise and reading) were used as intra group variables, and paired t-test was used to analyze BDNF index and behavior index (go reaction time and correct rate, Nogo correct rate); the average peaks of N2 and P3 in ERP data were analyzed by three factor repeated measurement ANOVA of 2 (intervention factors: exercise and reading) $\times 2$ (test tasks: go, Nogo) $\times 4$ (poles: $\mathrm{F}_{\mathrm{Z}}, \mathrm{FC}_{\mathrm{Z}}, \mathrm{C}_{\mathrm{Z}}, \mathrm{CP}_{\mathrm{Z}}$ ) Greenhouse geisse: was used to correct the statistical variables of dissatisfaction football shape test, and Bonferroni method was used for post comparison. The difference was significant $(\mathrm{P}<0.05)$. 
Article History: Received: 20 October 2020, Revised: 15 November 2020, Accepted: 08 December 2020, Publication: 28 February 2021

\section{RESULT}

\subsection{Sequential effect test}

Taking the response time and accuracy of Go task, the accuracy of Nogo task and bndf index as dependent variables, paired t-test was carried out on the scores of the two groups in the two tests (namely, exercise group a vs. exercise group B, reading group A vs. reading group B). The results showed that there was no significant difference between the two scores $(\mathrm{P}>0.05)$. Therefore, there is no order effect in the cross design of this study, and the corresponding data are combined to get the results of each period.

\subsection{EEG results}

In the go/nogo task, there are differences in ERP wave patterns induced by different regions of cerebral cortex under Go and Nogo conditions. Both groups induced negative wave N2 in 200-320 ms and positive wave P3 in 320-440 ms. See Figure 2.
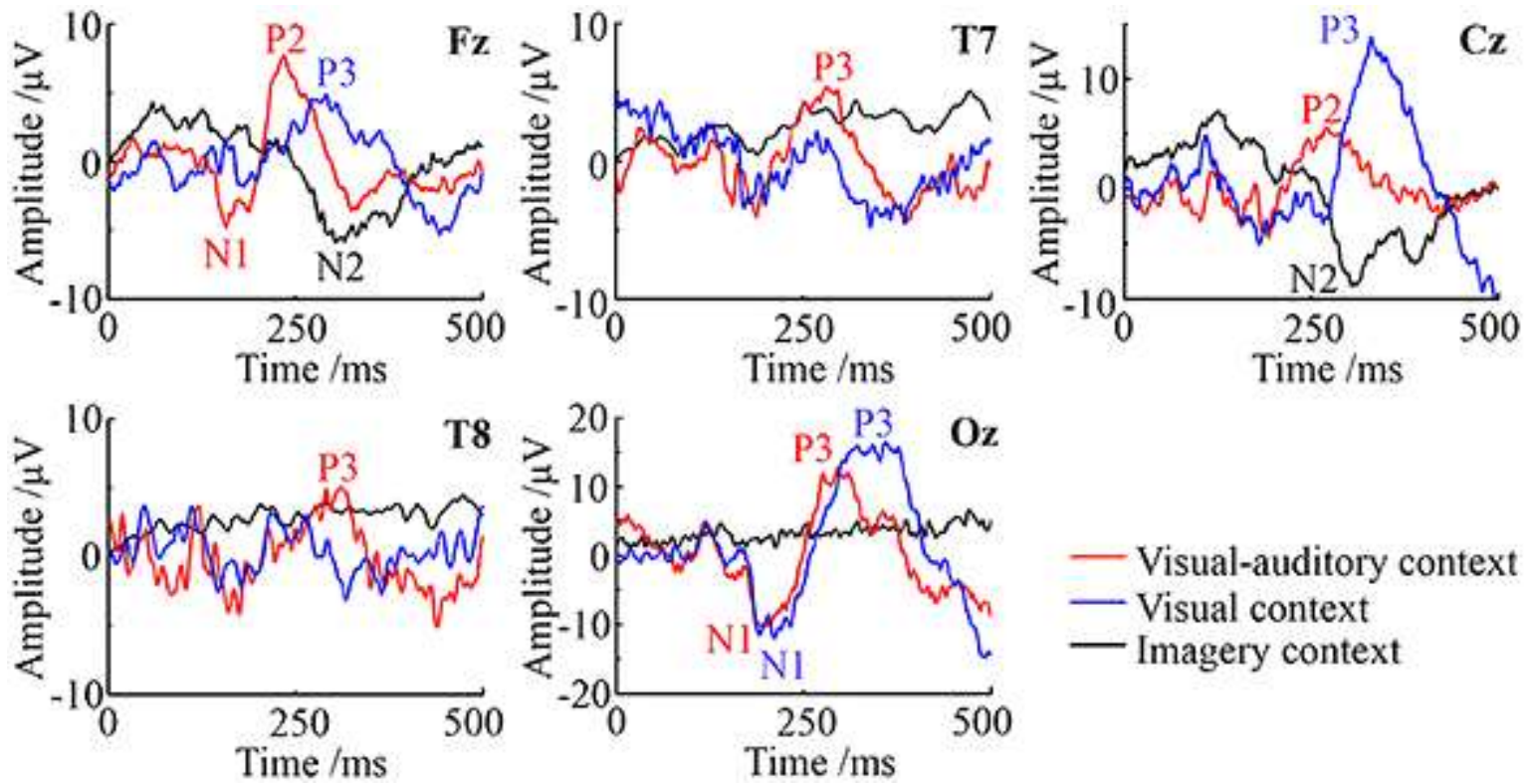

- Visual-auditory context

- Visual context

- Imagery context

Fig 2: ERP waveforms of Go condition (left) and Nogo condition (right) $\mathrm{C}_{Z}, \mathrm{FC}_{Z}$ points

3.3 Behavioral outcomes

The results of paired t-test with group as independent variable, reaction time, accuracy of go task and accuracy of Nogo task as dependent variable showed that the accuracy of go task in both groups was higher than $95 \%$, and exercise intervention was significantly higher than reading intervention $[\mathrm{t}(32)=2.522, \mathrm{P}=$ 0.017]. There was no significant difference in response time of go task between groups [t $(32)=-0.925, \mathrm{P}=$ 0.362]; the inhibition success rate of exercise intervention was significantly higher than that of reading intervention $[[\mathrm{t}(32)=9.520, \mathrm{P}<0.001]$. See Table 3 .

TABLE III. Comparison of Go Task Reaction Time and Nogo Task Correct Rate between

Exercise Intervention and Reading Intervention

\begin{tabular}{|c|c|c|c|c|}
\hline INDEX & $\begin{array}{c}\text { EXERCISE } \\
\text { INTERVENTION } \\
(\mathbf{N = 1 7 )}\end{array}$ & $\begin{array}{c}\text { READING } \\
\text { INTERVENTION } \\
(\mathbf{N = 1 7 )}\end{array}$ & $\mathbf{T}$ & $\mathbf{P}$ \\
\hline GO RT(MS) & $338.98 \pm 39.49$ & $352.81 \pm 47.28$ & -0.925 & 0.362 \\
\hline GO ACCURACY $(\%)$ & $97 \pm 3$ & $95 \pm 2$ & 2.575 & 0.018 \\
\hline
\end{tabular}




\begin{tabular}{|l|l|l|l|l|} 
ACCURACY OF NOGO (\%) & $75 \pm 8$ & $50 \pm 8$ & 9.520 & 0.000 \\
\hline
\end{tabular}

\section{DISCUSS}

4.1 acute aerobic exercise intervention increases the behavior performance of inhibition control of college students

Through the cognitive behavior test of inhibition ability, it is found that acute aerobic exercise has no significant effect on the reaction time of college students when they complete inhibition control task. It shows that moderate intensity aerobic exercise has no effect on the general perception ability of college students. This is consistent with previous studies. Dietrich's research on healthy people also did not find the improvement of perceptual response time. In the long-term aerobic exercise, it also showed the same effect as acute moderate intensity aerobic exercise. For patients with brain damage, the effect of long-term aerobic exercise could not be reflected in perceptual ability. But different from reaction time, after acute aerobic exercise, the correct rate of inhibition task was significantly improved. Acute aerobic exercise can effectively promote the general inhibition ability of college students. This result has also been confirmed by previous studies on healthy population, elderly population and brain damaged population. Therefore, the enhancement of inhibition ability of college students after aerobic exercise can effectively enhance their self-control ability, and then reduce the occurrence of bad habits of college students. In conclusion, we believe that acute aerobic exercise can effectively improve the inhibition ability of ordinary college students.

\subsection{Acute exercise intervention increases BDNF content in serum of College Students}

In order to explore the relationship between the inhibition ability of acute aerobic exercise and BDNF in peripheral serum of college students, we measured the BDNF in serum of college students after exercise intervention by ELISA. The results showed that acute aerobic exercise significantly increased the serum BDNF concentration of college students (more than 3 times of reading intervention), and was positively correlated with the improvement of inhibition ability. It is suggested that the increase of BDNF in peripheral blood serum may be related to the increase of inhibition ability of acute aerobic exercise. This study found that the BDNF content did not change significantly when RPE was 10 (relaxed), but changed only when RPE was 14 (a little hard). This suggests that the improvement of cognition depends on the intensity of exercise. In this experiment, we used $60 \%$ - 69\% of the maximum heart rate of moderate exercise intensity, the results showed that the exercise significantly increased the serum BDNF content in vitro. Exercise induced peripheral serum BDNF may affect the synaptic plasticity of mesolimbic system, promote cognitive function, improve executive function, and improve inhibition. Our experimental results provide a research idea for the BDNF hypothesis of exercise promoting inhibition in the future. At the same time, it should be pointed out that our results only show that there is a correlation between exercise inhibition and the increase of BDNF, but can not explain the causal relationship between the two. Future studies can verify whether BDNF is the main way to improve exercise inhibition by adding BDNF blockers in animal experiments or using gene knockout animals.

4.3 Acute aerobic exercise intervention increases the electrophysiological performance of inhibition control in College Students

After the intervention of acute aerobic exercise, N2 component reflects the corresponding reaction of behavior inhibition. The amplitude of N2 induced by Nogo task is larger than that of Go task, and the 
amplitude of $\mathrm{N} 2$ induced by activity condition is also larger than that of reading condition. Therefore, the amplitude of $\mathrm{N} 2$ is relatively increased under exercise and Nogo conditions. This result is consistent with previous studies. Folstein et al. found that N2 amplitude induced by anterior cortex was highly positively correlated with Nogo response. However, the change of N2 amplitude induced by Nogo stimulation is also considered as the dominant index of response to inhibition function. Therefore, the amplitude of Nogo-N2 in frontal area is often considered as an effective index to reflect the inhibition ability, and aerobic exercise can improve the inhibition ability, which is related to the inhibition of the activation of brain areas such as dorsolateral prefrontal cortex.

Except for N2 component, the difference of P3 component was reflected in the peak and latency. In the motor task, the P3 component was significantly larger than that in the reading task, which was consistent with the P3 effect observed in previous studies. The increase of P3 amplitude after acute aerobic exercise reflects that acute aerobic exercise increases the allocation of attention resources. At the same time, the source of P3 component is located in the right posterior part of the middle cingulate cortex, which is different from N2 component. Therefore, compared with the N2 component, the P3 component reflects the late stage of inhibitory processing. Hillman et al reported that acute aerobic exercise can induce the increase of P3 amplitude in prepubertal children, which may represent that individuals allocate more attention resources to this task. Moderate intensity aerobic exercise can not only cause lasting morphological changes in the brain, but also cause immediate chemical changes in the brain, so as to improve the arousal level of the brain and enhance the cognitive performance of the exerciser.

\section{CONCLUSION}

Acute aerobic exercise can effectively promote the inhibition ability of ordinary college students, and get support in behavior performance, physiological response and electrophysiological response. At the same time, the mechanism of acute aerobic exercise on inhibition ability lies in the rapid recognition of inhibition signal and the allocation of cognitive resources.

\section{ACKNOWLEDGEMENTS}

This work was supported by 2017 Qingdao social science planning research project (No. QDSKL1701029) and the Fundamental Research Funds for the Central Universities (No 201715019).

\section{REFERENCES}

[1] Deng Yunlong. Basic Idea of "rhythm Alternation Theory" in Sports Training. Journal of Shenyang Institute of Physical Education, 2007 (03): 7-11

[2] Tian Maijiu. New Development of Theoretical System of Sports Training in China. Journal of Beijing Sport University (2): 145-148

[3] Tian Ye, Wang Qing, Feng Lianshi. Scientific Monitoring and Adjustment of Competitive State of Elite Athletes. Sports Science, 2008, 28 (009): 3-11

[4] Zhang Aifang, Feng Weiquan. Effects of Chinese Herbal Medicine on Some Biochemical Indexes of Exercise Training Mice. Journal of Beijing Sport University, 2010 (03): 70-81 
[5] Zhan Jianguo. Development Status of Russian Competitive Sports and Experience and Enlightenment of Sports Training. Journal of Beijing Sport University, 2004 (06): 840-842

[6] Chen Xiaoping. on Special Characteristics -- the Main Problems and Countermeasures of Current Sports Training in China. Sports Science, 2007, 027 (002): 72-78

[7] Xu Benli. from Special Theory to Sports Training Science -- on the Formation and Development Trend of Sports Training Scientific Theory. Journal of Beijing Sport University, 2004 (06): 721-726

[8] Cao Jingwei, Yuan Shoulong, Xi Yi. Zhongguoliu in the Theoretical Research of Sports Training. Sports Science, 2004, 024 (002): 29-32

[9] Lu Changfen, Wang Jian. the Reform of the Curriculum System of Sports Training in China from the Perspective of Curriculum Reform of American Sports Colleges and Departments. Journal of Xi'an Institute of Physical Education, 2003, 20 (001): 96-100

[10]Zhan Jianguo, Chai Guorong, Yang Wenwen. from the Perspective of the 27th Olympic Games, the Strength Pattern of International Athletics and the Direction of China's Track and Field Training. Journal of Beijing Sport University, 2000, 023 (004): 540-543 